\title{
Diferencias de la conciliación vida-carrera en mujeres de Sonora y Coahuila, México*
}

\section{Differences in life-job balance among women of Coahuila and Sonora, Mexico}

\author{
Alicia Hernández Montaño** \\ José González Tovar \\ Dariela Janet Regino Rico \\ Universidad Autónoma de Coahuila \\ Elba Abril Valdez \\ María José Cubillas Rodríguez \\ Alejandra Córdova Moreno \\ Sandra Domínguez Ibáñez \\ Centro de Investigación en Alimentación y \\ Desarrollo, A. C., México \\ Recibido: 12 de agosto de 2016 \\ Revisado: 13 de septiembre de 2016 \\ Aceptado: 3 de noviembre de 2016
}

\section{Resumen}

El objetivo de este estudio fue analizar las diferencias de la conciliación de los componentes vida y carrera laboral de mujeres trabajadoras de dos estados del norte de México, Coahuila y Sonora, con el fin de conocer los contrastes en la distribución y la armonización de las tareas dentro y fuera de casa. Se aplicó una escala para medir la conciliación vidatrabajo a 556 mujeres en ambos estados. Se exploraron seis dimensiones: gratificación, interferencia, pareja y crianza, cuidado y salud, responsabilidad en el hogar y tiempo libre. Los resultados indican que la muestra de Sonora presenta niveles más altos de conciliación. En general, la incorporación de las mujeres al ámbito laboral les genera gratificaciones personales, sin embargo, esta condición no ha modificado, en lo substancial, la división sexual del trabajo, es decir, las mujeres cumplen con sus jornadas de trabajo y continúan en casa con las tareas de cuidado y atención de la familia.

Palabras clave: mujeres, género, conciliación vidatrabajo, familia. 


\section{Abstract}

The aim of this study was to analyze the differences in the life-job balance of women in two northern states of Mexico, Coahuila and Sonora, in order to know the contrasts in the distribution and harmonization of tasks inside and outside the home. A scale was applied to measure life-job balance to 556 women in both states. Six dimensions were explored: gratification, interference, family and parenting, health care and responsibility at home and free time. The results indicate that the sample of Sonora has higher levels of conciliation. In general, the incorporation of women into the workplace generates personal rewards, however, this condition has not substantially changed the sexual division of labor, and women working their daily hours and then continue with care and family care tasks at home.

Keywords: women, gender, life-job balance, family.

\section{Introducción}

En las últimas décadas la intervención de las mujeres en el mercado productivo se ha incrementado sustancialmente. A partir del 2006 su tasa de participación en el mundo ha alcanzado 67 mujeres por cada 100 hombres (Organización Internacional del Trabajo - OTI, 2007). Este aumento no ha sido privativo para las mujeres de México, pues se estima que actualmente el $38 \%$ participa en el sector laboral (Instituto Nacional de Estadística y Geografía - INEGI, 2013).

Para el presente estudio nos enfocaremos en dos estados del norte de México: Coahuila y Sonora. Como se muestra en la tabla 1 , son entidades que presentan diferencias en su contexto laboral. Para el caso de Coahuila, la participación económicamente activa (PEA) de las mujeres es de 37.1 $\%$, mientras que para Sonora es de $40.1 \%$, esta última por encima de la media nacional. Si bien su participación en las actividades económicas ha tendido a aumentar gradualmente, muchas de ellas se ocupan en actividades de baja productividad y remuneración. En ambos estados, cerca del $34 \%$ de la PEA ocupada percibe dos salarios mínimos o menos; en el caso de las mujeres esta proporción es del $41 \%$. De acuerdo al nivel de instrucción, la proporción de mujeres con mayor representatividad en el campo laboral son: para Sonora, las de secundaria y media superior (43\%), seguidas por las que tienen estudios superiores (40.6\%); para el caso de Coahuila es similar, solo que varían un poco los porcentajes, $38 \%$ y 38.4 $\%$ respectivamente. El comercio es la actividad económica donde se ocupa la mitad de las mujeres trabajadoras de ambos estados (Secretaria del Trabajo y Previsión Social - STPS, 2016).

Respecto a otras características sociodemográficas encontramos que, en cuestiones de educación, las mujeres de Coahuila tienden a estudiar en promedio 0.5 años más que las de Sonora, además de presentar un índice menor de analfabetismo. Sin embargo, las sonorenses tienen mayor representatividad en puestos políticos de toma de decisiones, muy por encima de las coahuilenses (INEGI, 2013).

Ambos estados presentan altos índices de violencia contra las mujeres, empero, Coahuila está dentro de los estados, a nivel nacional, con una de las más altas tasa de homicidios contra las mujeres (INEGI, 2013). 
Tabla 1.

Porcentajes del contexto laboral de los estados de Sonora y Coahuila, primer trimestre 2016.

\begin{tabular}{lcc}
\hline Categoría & Sonora & Coahuila \\
\hline Contexto laboral & & \\
\hline Participación laboral (PEA) mujeres de 14 años y más* & 40.1 & 37.1 \\
\hline Ocupación por nivel de educación & & \\
\hline Sin instrucción & 27.6 & 28.7 \\
Primaria & 32.8 & 33.6 \\
Secundaria y media superior & 43.0 & 38.0 \\
Superior & 40.6 & 38.4 \\
\hline Ocupación por rama de actividad económica & & \\
\hline Actividades agropecuarias & 18.7 & 9.2 \\
Industria manufacturera & 44.6 & 28.3 \\
Industria extractiva y electricidad & 8.6 & 10.9 \\
Construcción & 7.1 & 1.2 \\
Comercio & 56.1 & 50.6 \\
Transportes y comunicación & 13.6 & 15.3 \\
Otros servicios & 50.5 & 53.8 \\
Gobierno y organismos internacionales & 39.8 & 47.0 \\
No especificado & 25.3 & 28.6 \\
\hline
\end{tabular}

*La media nacional es de 38 \%. Fuente: Secretaría del Trabajo y Previsión Social (2016).

Tabla 2.

Porcentajes de las características del contexto sociodemográficas de los estados de Sonora y Coahuila, 2013 y 2014.

\begin{tabular}{lcc}
\hline Categoría & Sonora & Coahuila \\
\hline Escolaridad* & & 9.4 años \\
\hline Promedio mujeres de 15 años y más & 8.9 años & 2.6 \\
\hline Tasa de analfabetismo en mujeres de 15 años y más & 3.7 & $5 \%$ \\
\hline Nupcialidad* & 58.0 & 61.2 \\
\hline Casada o unión libre & 15.6 & 14.0 \\
Separada, divorciada o viuda & 24.7 años & 23 años \\
\hline Edad promedio de la primer unión de mujeres de 15 años y más & & 12 \\
\hline Participación política** & 24.2 & 2.6 \\
\hline Poder legislativo & 11.1 & \\
Poder ejecutivo & & \\
\hline Violencia por condición de género* & 68.1 & 7.1 \\
\hline Mujeres de 15 años y más con un incidente de violencia en la & 3.9 & \\
pareja, espacio laboral, comunitario o escolar & & \\
\hline Tasa de muertes por homicidio & & \\
\hline
\end{tabular}

*Tasa de 2013. **Tasa 2014. Fuente: INEGI, 2013 y 2015.

Independientemente del nivel de formación académica, más mujeres con responsabilidades familiares e hijos menores buscan trabajo fuera del hogar, algunas como vía para un crecimiento personal y otras para lograr subsistir. Esta realidad social ha generado la necesidad de armonizar las cargas de trabajo que surgen del contexto laboral y las tareas de casa.

El concepto "reconciliación" dentro de este contexto temático se refiere a la necesidad de armonizar el tiempo dedicado al trabajo y la vida 
familiar (Tena, 2013), un problema que ha sido definido principalmente femenino. Cuenta de esto, son las políticas laborales que plantean "estrategias” que permitan a las mujeres continuar con su vida laboral sin descuidar sus responsabilidades familiares, planteamientos que refuerzan la idea de las mujeres como amas de casa y cuidadoras (Faur, 2006).

Un aspecto importante del equilibrio laboral-personal es el número de horas que una persona trabaja. La Organización para la Cooperación y el Desarrollo Económico (OCDE) sugiere que un horario de trabajo largo puede resultar perjudicial para la salud, poner en peligro la seguridad y aumentar el estrés. En México el $35 \%$ de los hombres trabajan con un horario muy largo, en comparación con el $18 \%$ del caso de las mujeres (OCDE, 2015). Sin embargo, estas últimas invierten más tiempo en las tareas del hogar y crianza de los hijos, lo que hace que la mayoría de ellas finalmente cumplan con jornadas largas de trabajo.

Esta situación se ve reflejada en los resultados estadísticos a nivel nacional (Encuesta Nacional de Ocupación y Empleo, 2012). El 36.8 \% de las mujeres de 14 años y más, forman parte de la población económicamente activa (PEA), de las cuales el 94.5 $\%$ combina sus actividades laborales con los quehaceres domésticos. La distribución por sexo de la población que realiza trabajo no remunerado y de cuidados muestra que el 52.8 \% está constituido por mujeres y el $47.2 \%$ por hombres. Sin embargo, cuando se analiza el número de horas trabajadas y el valor económico generado, las diferencias por sexo aumentan significativamente: las mujeres aportan el $78.3 \%$ de las horas destinadas a labores en el hogar y generan el $76.1 \%$ de valor económico; mientras que los hombres contribuyen con el $21.7 \%$ de horas, equivalente al $23.9 \%$ del valor económico (INEGI, 2014). Aun así, la mujer tiene 77 años de esperanza de vida contra 74 años de los hombres. Sin embargo, el que las mujeres sobrevivan más años no significa que su calidad de vida también sea mayor, pues en la etapa de su vejez no todas cuentan con seguridad social, haciéndose dependientes de su descendencia que aprovechan su mano de obra cuando aún pueden ser útiles para cuidar de la casa y los nietos mientras la hija trabaja.
La exigencia de hacer compatibles el trabajo extradoméstico con el cuidado de la familia parece conllevar tres principales tipos de conflicto (Martínez, Vera, Paterna y Alcázar, 2002). En primer lugar, está lo que compete con el tiempo, es decir, las tareas domésticas exigen tiempo completo, son cíclicas que demandan al menos ocho horas diarias de trabajo continuo. En segundo lugar, surgen las problemáticas relacionadas con la tensión y el agotamiento, la sobrecarga de trabajo que involucra el llevar ambos escenarios y además la exigencia de llevarlo de forma "exitosa" repercutiendo en una tensión y estrés emocional continuo. Finalmente, el tercero se vincula con la presencia de conductas y expectativas que se vuelven incompatibles, es decir, desde el punto de vista social, se espera que las mujeres sean "buenas madres" y "buenas esposas", actividades que requieren, de acuerdo con los estereotipos de género, tiempo completo ante las tareas del hogar y el cuidado de los hijos, poniéndolas ante una encrucijada si estas optan por trabajos extra domésticos, pues la conciliación de ambos escenarios se dificulta generando malestares y enfermedad en las mujeres.

En este sentido, los estereotipos de género son un conjunto de creencias populares sobre las actividades, los roles, los rasgos, las características o atributos que caracterizan y distinguen a los hombres de las mujeres (Moya, 2003). Estos significados están conformados por reglas, obligaciones, características, comportamientos, prohibiciones, expectativas y valores que son transmitidos y reforzados en las personas como parte de su proceso de identidad para aprender a ser hombres y mujeres (Rocha y Díaz-Loving, 2012).

De esta manera, a las mujeres se les ha asociado con mayor regularidad a características como la sensibilidad, el amor, la complacencia, la entrega, la fidelidad y el romanticismo (Díaz, 2012; DíazLoving, Rivera y Sánchez, 2001), estereotipos idóneos para responsabilizar y justificar sus labores de maternidad y convivencia en el ámbito familiar.

Por otro lado, los estereotipos asociados a lo masculino son de corte instrumental encaminados al logro, como la competencia, la toma de decisio- 
nes, la inteligencia, la firmeza, el atrevimiento y la autosuficiencia (Díaz, 2012; Díaz-Loving et al., 2001). No se les asocia con la expresión de los afectos, la paciencia, la delicadeza y la ternura, mismas que justifican su desapego en la crianza y cuidado de los hijos (Bonino, 2004; Ortega, 1998; Tena y Jiménez, 2006).

Esto nos ayuda a comprender que la "especialización" de las mujeres en el campo reproductivo y la del varón en el campo productivo, se debe a una serie de creencias culturales que nos hacen suponer que cada uno de estos actores posee ciertas cualidades o habilidades "innatas" con base en el sexo biológico al que pertenecen.

De ahí, que la división más básica es la que separa el trabajo reproductivo del trabajo productivo. El primero de ellos, donde las mujeres tienen un papel protagónico, son acciones vinculadas al cuidado de la familia y a lo doméstico; carece de un reconocimiento social, son actividades cíclicas y cotidianas, no tiene horarios, vacaciones, no es remunerado económicamente y se realizan a lo largo de todo el ciclo de la vida. El segundo, el trabajo no doméstico o productivo, un espacio tradicionalmente masculino, son acciones vinculadas con la producción y la política, cuenta con un reconocimiento social, producen bienes o servicios y tienen un valor de cambio. Se da dentro de un horario específico, generalmente en la semana, con descansos el fin de semana y se efectúa hasta alcanzar la jubilación (Benería, 2006).

Por tanto, podemos afirmar que las construcciones de género no solo implican la asignación de tareas diferentes a hombres y mujeres, sino que establecen y fomentan desigualdad e injusticia, con mayor impacto y desventaja para las mujeres.

En este sentido, el género como categoría de análisis, permite en la investigación descubrir e interpretar las relaciones de género atendiendo a los elementos culturales y sociales. En el caso del estudio de la conciliación vida-trabajo permite explicar las diferencias en la distribución y la armonización de las tareas dentro y fuera de casa que llevan a cabo mujeres con trabajo remunerado y que explican de alguna manera nuestro problema de investigación.

\section{Método}

\section{Participantes}

Se incluyeron a 554 mujeres, el criterio de selección mediante un muestreo intencional, tomó en cuenta que las participantes actualmente desempeñaran una actividad laboral remunerada, formal o informal. Las mujeres se seleccionaron de dos ciudades, de dos estados diferentes de la república mexicana, 419 casos de la ciudad de Saltillo, Coahuila y 135 casos de la ciudad de Hermosillo, Sonora. La edad promedio de las mujeres fue de 39 años, con una desviación típica de 11 . La edad mínima fue de 18 y la máxima presente fue 66 años. En cuanto al estado civil y conyugal, el $45.8 \%(n=254)$ de las mujeres dijo que es madre soltera; $27.8 \%(n=154)$ se encuentran casadas; $14 \%(n=82)$ son divorciadas; $8.5 \%(n=$ 47) están solteras y el $1.8 \%(n=10)$ dijo vivir en unión libre. El $36.5 \%(n=202)$ de la muestra tiene estudios universitarios; el $20.4 \%(n=113)$ tiene alguna carrera técnica; $16.6 \%(n=92)$ estudió hasta preparatoria o bachillerato; $12.8 \%(n=71)$ cuenta con secundaria; $4.2 \%$ concluyó solo la educación primaria $(n=23)$; solo el $9.4 \%(n=52)$ reportó estudios de posgrado.

\section{Instrumento}

Se utilizó la Escala de Conciliación Vida-Trabajo (Hernández y González, 2015), misma que contiene 25 afirmaciones agrupadas en seis dimensiones: gratificación ( $\alpha=.851)$, interferencia $(\alpha=.841)$, pareja y crianza ( $\alpha=.695)$, cuidado y salud ( $\alpha=831)$, responsabilidad en el hogar $(\alpha=.751)$ y tiempo libre $(a=.684)$. El formato de respuesta fue mediante una escala ordinal de frecuencias que va de Nun$\mathrm{ca}=0$ hasta Siempre $=4$. El instrumento obtuvo un índice de fiabilidad, medido a través del coeficiente alfa de Cronbach de $\alpha=.874$.

\section{Procedimiento}

La recolección de datos tuvo lugar en dos ciudades del norte de México: Sonora y Saltillo. Se asistió a los centros de trabajo de las entrevistadas, dentro 
de sus horarios laborales, recabando la información de forma individual y en formato de autoreporte. El análisis de los datos se realizó mediante las frecuencias de las características laborales y familiares de las participantes, posteriormente se realizó una prueba de hipótesis contrastando las dimensiones de la conciliación entre vida y carrera a partir de la ciudad a la que pertenecían las mujeres, se utilizó para tal efecto una prueba $t$ para muestras independientes.

\section{Resultados y discusión}

Se obtuvieron porcentajes por estado de las características laborales y familiares básicas de las mujeres que participaron en el estudio, de manera que se obtuviera una descripción de las condiciones en la cuales las mujeres se han incorporado al sector productivo. En el caso de las mujeres de Coahuila, el $47 \%$ se desempeñan en el sector público, el $43 \%$ en el sector privado y solo el $8 \%$ en organismos no gubernamentales; en contraste en Sonora, el $80 \%$ de las mujeres entrevistadas pertenecen al sector público, el $16 \%$ al sector privado y solo el $1.5 \%$ a organismos no gubernamentales.

En Coahuila, el $70 \%$ de las mujeres reporta que recibe ayuda para las labores de la casa y el $66 \%$ recibe apoyo con el cuidado de los hijos, esto para cumplir con las jornadas laborales, en contraste el 33 \% de las mujeres de Sonora recibe apoyo para las labores del hogar y el $66 \%$ recibe apoyo para el cuidado de los hijos. En promedio, las mujeres coahuilenses laboran 8 horas diarias y tienen el beneficio de 27 días de vacaciones al año en promedio, las mujeres de Sonora laboran una hora más y también reciben en promedio 27 días de vacaciones.

Por otro lado, para identificar las diferencias en las dimensiones de la conciliación entre la vida familiar y laboral de las mujeres se procesó la prueba $t$ para muestras independientes, se realizó el contraste entre Coahuila y Sonora. El nivel de significancia fue de $p \leq .010$. Se encontraron diferencias significativas en todos los componentes que integran el factor de gratificación; la muestra correspondiente a la ciudad de Hermosillo, Sono-
Tabla 3.

Características laborales y familiares de las mujeres participantes por estado.

\begin{tabular}{lccc}
\hline \multirow{2}{*}{ Indicador } & \multirow{2}{*}{ Categoría } & \multicolumn{2}{c}{ Valor por estado } \\
\cline { 2 - 4 } & Coahuila & Sonora \\
\cline { 2 - 4 } Área laboral & Sector privado & $43.9 \%$ & $16.3 \%$ \\
\cline { 2 - 4 } & $\begin{array}{c}\text { Organismos no } \\
\text { gubernamentales }\end{array}$ & $8.6 \%$ & $1.5 \%$ \\
\hline $\begin{array}{l}\text { Apoyo para } \\
\text { labores de } \\
\text { la casa }\end{array}$ & Sí & $70.7 \%$ & $33.3 \%$ \\
\hline $\begin{array}{lccc}\text { Apoyo para } \\
\text { el cuidado } \\
\text { de los hijos }\end{array}$ & Sí & $29.3 \%$ & $66.7 \%$ \\
\cline { 2 - 4 } $\begin{array}{l}\text { Promedio de horas por jornada } \\
\text { laboral }\end{array}$ & $66.3 \%$ & $44.4 \%$ \\
\hline $\begin{array}{l}\text { Promedio de días de vacaciones } \\
\text { al año }\end{array}$ & 27.64 & 27.20 \\
\hline \begin{tabular}{l} 
Promedio de número de hijos \\
\hline
\end{tabular} & 3.03 & 2.22 \\
\hline
\end{tabular}

Fuente: elaboración propia.

ra encuentra en el trabajo una oportunidad para aprender cosas que les sirvan en su cotidianidad y fomentar las amistades. La variable que mide la sensación de utilidad, es la que tiene la media más cercana al valor máximo de la escala. Es decir, el trabajo no solo aumenta sus conocimientos, habilidades, aptitudes o actitudes, dentro y fuera de su entorno de trabajo, sino que incrementa su sentido de legitimidad al sentirse reconocidas por su entorno.

Estas experiencias pueden representar algunas de las ventajas de trabajar fuera de casa, pues les permite contar con una red de apoyo social y un espacio de esparcimiento y diversión. Contar con su propio recurso económico les da una sensación de independencia al poder elegir salir con amigas o comprarse algo personal.

Sin embargo, hay que destacar que ambas muestras presentaron promedios que no superan los 3 puntos que representa una frecuencia intermedia. 
Tabla 4.

Contraste mediante la prueba t para muestras independientes del componente de gratificación.

\begin{tabular}{|c|c|c|c|c|c|c|}
\hline Variable dependiente & Estado & M & DT & $\mathrm{t}$ & gl & $\mathrm{p}$ \\
\hline \multirow{2}{*}{ Capacitarme constantemente* } & Coahuila & 1.69 & 1.078 & -4.589 & 489 & .000 \\
\hline & Sonora & 2.20 & 1.104 & -4.538 & 231.254 & .000 \\
\hline \multirow{2}{*}{$\begin{array}{l}\text { Aprender cosas que me sirvan } \\
\text { en mi vida diaria }\end{array}$} & Coahuila & 1.65 & 1.170 & -7.834 & 548 & .000 \\
\hline & Sonora & 2.53 & 1.057 & -8.252 & 249.417 & .000 \\
\hline \multirow{2}{*}{ Sentirme útil y reconocida* } & Coahuila & 2.06 & 1.063 & -7.035 & 533 & .000 \\
\hline & Sonora & 2.79 & .980 & -7.323 & 248.309 & .000 \\
\hline \multirow{2}{*}{$\begin{array}{l}\text { Ampliar mi círculo de } \\
\text { amistades }\end{array}$} & Coahuila & 1.57 & 1.161 & -6.862 & 551 & .000 \\
\hline & Sonora & 2.33 & .970 & -7.520 & 268.346 & .000 \\
\hline \multirow{2}{*}{$\begin{array}{l}\text { Mantener una relación cercana } \\
\text { con amigos }\end{array}$} & Coahuila & 1.68 & 1.147 & -7.029 & 533 & .000 \\
\hline & Sonora & 2.44 & .912 & -7.866 & 287.914 & .000 \\
\hline \multirow{2}{*}{$\begin{array}{l}\text { Tener una relación cercana con } \\
\text { mis compañeros de trabajo }\end{array}$} & Coahuila & 1.77 & 1.056 & -9.573 & 551 & .000 \\
\hline & Sonora & 2.73 & .868 & -10.578 & 272.846 & .000 \\
\hline \multirow{2}{*}{$\begin{array}{l}\text { Contar con recurso económico } \\
\text { para realizar actividades } \\
\text { recreativas o culturales* }\end{array}$} & Coahuila & 1.61 & 1.042 & -7.769 & 552 & .000 \\
\hline & Sonora & 2.42 & 1.096 & -7.569 & 217.467 & .000 \\
\hline
\end{tabular}

*Se asumieron varianzas iguales, $M=$ media, gl=grados de libertad, $D T=$ desviación típica, $p=$ nivel de significancia. Fuente: elaboración propia.

La dimensión de interferencia también mostró diferencias significativas en todos los componentes que lo integran, presentándose un menor nivel de conciliación para las mujeres de Coahuila. Para estas, la vida laboral dificulta su cuidado personal, particularmente el cuidado de su imagen y su alimentación, al no tener tiempo suficiente para planear las compras y la preparación de alimentos equilibrados. Hay que tomar en cuenta que esta población, en comparación con la de Sonora, tiene en promedio un hijo más y el $43.9 \%$ trabaja en el sector público, espacio donde los horarios de trabajo suelen requerir, en grandes ocasiones, de tiempo más allá de su horario habitual, por lo que dichas condiciones pudieran representar un obstáculo para la conciliación en este rubro.

Tabla 5.

Contraste mediante la prueba t para muestras independientes del componente de interferencia.

\begin{tabular}{|c|c|c|c|c|c|c|}
\hline & Estado & Media & Desviación típ. & $\mathrm{t}$ & $\mathrm{gl}$ & $\mathrm{p}$ \\
\hline \multirow{2}{*}{$\begin{array}{l}\text { Tiempo suficiente para el cuidado de mi ima- } \\
\text { gen personal* }\end{array}$} & Coahuila & 1.97 & .955 & -9.470 & 552 & .000 \\
\hline & Sonora & 2.85 & .910 & -9.707 & 236.445 & .000 \\
\hline \multirow{2}{*}{$\begin{array}{l}\text { Tiempo suficiente para el cuidado de mi hi- } \\
\text { giene personal }\end{array}$} & Coahuila & 2.43 & .870 & -13.322 & 552 & .000 \\
\hline & Sonora & 3.52 & .679 & -15.107 & 287.724 & .000 \\
\hline \multirow{2}{*}{$\begin{array}{l}\text { Contar con recurso económico para comprar- } \\
\text { me algún artículo personal* }\end{array}$} & Coahuila & 1.94 & .995 & -9.513 & 549 & .000 \\
\hline & Sonora & 2.87 & .932 & -9.835 & 237.912 & .000 \\
\hline \multirow{2}{*}{$\begin{array}{l}\text { Dedicar tiempo suficiente para la prepara- } \\
\text { ción de alimentos equilibrados en casa (plato } \\
\text { del buen comer)* }\end{array}$} & Coahuila & 1.74 & 1.042 & -8.243 & 552 & .000 \\
\hline & Sonora & 2.57 & .966 & -8.568 & 242.285 & .000 \\
\hline \multirow{2}{*}{$\begin{array}{l}\text { Disponer de tiempo para planear las compras } \\
\text { de alimentos semanalmente* }\end{array}$} & Coahuila & 1.84 & 1.033 & -9.638 & 550 & .000 \\
\hline & Sonora & 2.81 & .986 & -9.870 & 236.530 & .000 \\
\hline
\end{tabular}

*Se asumieron varianzas iguales, $M=$ media, gl=grados de libertad, DT= desviación típica, $\mathrm{p}=$ nivel de significancia. Fuente: elaboración propia. 
Respecto al componente de pareja y crianza, también se encontraron diferencias significativas entre las mujeres de ambos estados, es decir, existe un nivel más alto de conciliación en la muestra de Coahuila. Reportan que sus parejas están de acuerdo en que trabajen fuera de casa, no teniendo conflictos por las actividades que se derivan de este. Además de que las horas de trabajo no interfieren con sus actividades familiares y viceversa. Lo que implica que estas mujeres presentan buenas condiciones de equilibrio entre su vida laboral y las características de su dinámica familiar y de pareja.

Cabe recordar que estas mujeres cuentan en más de la mitad de los casos con ayuda para las labores domésticas y el cuidado de los hijos, podríamos decir que se trata de una estrategia que permite a las mujeres continuar con su vida laboral sin descuidar sus responsabilidades familiares, planteamiento que, de acuerdo con Faur (2006), refuerza la idea de las mujeres como responsables mayoritarias de las tareas de casa.

Tabla 6.

Contraste mediante la prueba t para muestras independientes del componente de pareja y crianza.

\begin{tabular}{|c|c|c|c|c|c|c|}
\hline & Estado & Media & Desviación típ. & $\mathrm{t}$ & $\mathrm{gl}$ & $\mathrm{p}$ \\
\hline \multirow{2}{*}{$\begin{array}{l}\text { Existen conflictos con mi pareja por cuestiones } \\
\text { laborales (salidas tarde, viajes, exceso de } \\
\text { trabajo) }\end{array}$} & Coahuila & .74 & 1.009 & -.877 & 516 & .000 \\
\hline & Sonora & .83 & 1.007 & -.878 & 196.586 & .000 \\
\hline \multirow{2}{*}{$\begin{array}{l}\text { Mi pareja considera que sería mejor que me } \\
\text { dedicara a cuidar a los hijos y atender la casa en } \\
\text { vez de trabajar* }\end{array}$} & Coahuila & .62 & .878 & -1.217 & 515 & .000 \\
\hline & Sonora & .74 & 1.245 & -1.013 & 154.699 & .000 \\
\hline \multirow{2}{*}{$\begin{array}{l}\text { Las horas de trabajo interfieren con el tiempo } \\
\text { que debo dedicar a mis hijos y familia }\end{array}$} & Coahuila & .99 & .984 & -4.228 & 534 & .000 \\
\hline & Sonora & 1.41 & 1.003 & -4.185 & 219.155 & .000 \\
\hline \multirow{2}{*}{$\begin{array}{l}\text { Las horas que dedico a mis hijos y familia } \\
\text { interfieren con mi trabajo }\end{array}$} & Coahuila & .39 & .682 & -6.932 & 537 & .000 \\
\hline & Sonora & .90 & .925 & -5.967 & 183.222 & .000 \\
\hline \multirow{2}{*}{$\begin{array}{l}\text { He tenido que rechazar una promoción o } \\
\text { traslado laboral por razones familiares }\end{array}$} & Coahuila & .50 & .807 & -2.277 & 535 & .000 \\
\hline & Sonora & 70 & 1.110 & -1.943 & 178.313 & .000 \\
\hline
\end{tabular}

*Se asumieron varianzas iguales, $M=$ media, gl=grados de libertad, DT= desviación típica, $\mathrm{p}=$ nivel de significancia. Fuente: elaboración propia.

Respecto a la dimensión del cuidado de la salud, existen diferencias significativas en las 3 dimensiones que la integran. Las mujeres de Sonora presentan mayores niveles de conciliación en esta categoría, lo que representa una diferencia significativa con respecto a las mujeres de Coahuila. Es decir, consiguen armonizar su vida laboral con ac- tividades deportivas y una dieta balanceada para mantener su condición física. Al parecer, salir del ámbito privado promueve en las mujeres un autocuidado de su imagen y salud física, algo que pudiera estar relacionado con las exigencias del medio laboral y social por mantener una "buena presentación". 
Tabla 7.

Contraste mediante la prueba t para muestras independientes del componente de cuidado de la salud.

\begin{tabular}{lcccccc}
\hline & Estado & Media & Desviación típ. & $\mathrm{t}$ & $\mathrm{gl}$ & $\mathrm{p}$ \\
\hline \multirow{2}{*}{\begin{tabular}{l} 
Mantener una dieta balanceada \\
\cline { 2 - 7 }
\end{tabular}} & Coahuila & 1.59 & 1.041 & -8.577 & 527 & .000 \\
\hline $\begin{array}{l}\text { Tener tiempo para realizar alguna } \\
\begin{array}{l}\text { actividad física como deporte, zumba, } \\
\text { aerobics, baile, etc. }\end{array}\end{array}$ & Sonora & 2.47 & .997 & -8.763 & 235.803 & .000 \\
\hline & Coahuila & 1.32 & 1.153 & -7.279 & 552 & .000 \\
Mantener mi condición física & Coahuila & 1.41 & 1.095 & -7.304 & 549 & .000 \\
\cline { 2 - 7 } & Sonora & 2.21 & 1.140 & -7.154 & 219.826 & .000 \\
\hline
\end{tabular}

*Se asumieron varianzas iguales, $M=$ media, gl=grados de libertad, DT= desviación típica, $\mathrm{p}=$ nivel de significancia. Fuente: elaboración propia.

El componente de responsabilidad en el hogar muestra diferencias significativas en sus 3 elementos que la integran. Las mujeres de Sonora tienen mejores condiciones de armonía entre su vida laboral y la distribución de las tareas del hogar y cuidado de los hijos. Al parecer existe una aceptación positiva, por parte de los varones, ante la incorporación de sus parejas al ámbito laboral. Esto introduce nuevos roles para ellas, pasan a ser también proveedoras económicas de los hogares (Carmona, 2006), situación que puede responder a la actual necesidad del ingreso de dos sueldos para solventar los gastos de una familia.
Sin embargo, el que los varones estén de acuerdo con esta incorporación, no tiene un alto impacto en su participación de los quehaceres domésticos y el cuidado de los hijos de la forma en que ellas quisieran, pues el promedio no supera los 3 puntos que representa una frecuencia intermedia. Aun así, es más alto que en el caso de las mujeres de Coahuila.

Es claro, para esta población, una división sexual del trabajo donde las mujeres tienen un papel protagónico en las responsabilidades del hogar y cuidado de los hijos (Benería, 2006), situación que hace que cumplan con jornadas largas de trabajo, provocando posiblemente una carga de labor que afecte su bienestar.

Tabla 8.

Contraste mediante la prueba t para muestras independientes del componente de responsabilidad en el hogar.

\begin{tabular}{lcccccc}
\hline & Estado & Media & Desviación típ. & $\mathrm{t}$ & $\mathrm{gl}$ & $\mathrm{p}$ \\
\hline $\begin{array}{l}\text { Mi pareja está de acuerdo con } \\
\text { que trabaje* }\end{array}$ & Coahuila & 1.82 & 1.064 & -14.682 & 503 & .000 \\
\cline { 2 - 7 } $\begin{array}{l}\text { Los quehaceres de la casa están } \\
\text { distribuidos con mi pareja de la } \\
\text { forma que quisiera }\end{array}$ & Sonora & 3.46 & 1.076 & -14.595 & 196.895 & .000 \\
\cline { 2 - 7 } $\begin{array}{l}\text { Mi pareja se involucra en el } \\
\begin{array}{l}\text { cuidado de los niños de la forma } \\
\text { que quisiera* }\end{array}\end{array}$ & Coahuila & 1.15 & 1.043 & -8.373 & 522 & .000 \\
\hline & Coahora & 2.09 & 1.228 & -7.680 & 179.257 & .000 \\
\hline & Sonora & 1.28 & 1.114 & -11.200 & 515 & .000 \\
\hline
\end{tabular}

*Se asumieron varianzas iguales, $M=$ media, gl=grados de libertad, DT= desviación típica, $\mathrm{p}=$ nivel de significancia. Fuente: elaboración propia. 
Finalmente, se encontraron diferencias significativas para el componente tiempo libre. Las mujeres de Coahuila reportan que este no se ve interferido por sus actividades de trabajo ni cuestiones familiares. Sin embargo, no queda claro qué entienden por tiempo libre. Para Phail (1999), es un ámbito de posible autonomía donde el individuo escoge libremente lo que es grato, le recrea o le proporciona placer, empero, las mujeres eligen -y entienden como tiempo libre- actividades que involucran la recreación o cuidado de sus hijos, más allá de actividades que abonen a su descanso o recreación individual, razón por la cual este componente pudo presentar una alta conciliación en la vida de las participantes. Cabe señalar, que la magnitud de las diferencias no representa una brecha importante entre ambas muestras.

Tabla 9.

Contraste mediante la prueba t para muestras independientes del componente de tiempo libre.

\begin{tabular}{lcccccc}
\hline & Estado & Media & Desviación típ. & $\mathrm{t}$ & $\mathrm{gl}$ & $\mathrm{p}$ \\
\hline El tiempo que dedico al trabajo & Coahuila & .68 & .914 & -4.322 & 543 & .000 \\
\cline { 2 - 7 } interfiere con mi tiempo libre & Sonora & 1.10 & 1.107 & -3.921 & 193.492 & .000 \\
\hline El tiempo que dedico a la familia & Coahuila & .60 & .901 & -3.259 & 545 & .000 \\
\cline { 2 - 7 } interfiere con mi tiempo libre & Sonora & .91 & 1.138 & -2.895 & 188.152 & .000 \\
\hline
\end{tabular}

$M=$ =media, gl=grados de libertad, DT= desviación típica, $p=$ nivel de significancia. Fuente: elaboración propia.

\section{Conclusiones}

El objetivo de la presente investigación fue realizar un contraste de la conciliación de los componentes vida y carrera laboral de mujeres trabajadoras de Coahuila y Sonora. De acuerdo a los resultados, existen diferencias a favor de estas últimas, es decir, presentan niveles más altos de conciliación.

De 6 dimensiones que conforman la Escala de Conciliación Vida-Trabajo, 4 estuvieron a favor de las sonorenses: gratificación, interferencia, cuidado de la salud y responsabilidad en el hogar. Lo anterior sugiere que este grupo de mujeres cuentan con mejores recursos personales, sociales y quizá laborales para armonizar sus responsabilidades. A nivel estatal, tienen mayor porcentaje de escolarización y menor analfabetismo, pero tienen mayor representatividad en puestos políticos claves para la toma de decisiones (INEGI, 2013), lo que puede sugerir que es un contexto que favorece la participación positiva de las mujeres.

De manera general, trabajar fuera de casa tiene dos polos para las mujeres. Por un lado, les genera experiencias gratificantes que impactan direc- tamente en su desarrollo y cuidado personal, es decir, el campo laboral es un contexto enriquecido donde las mujeres pueden seguir preparándose personal y profesionalmente, lo que incrementa su sensación de utilidad. Esto puede responder a que históricamente el trabajo doméstico no remunerado ha tenido poco valor social al darse por sentado que es parte de las funciones "naturales" de las mujeres (Moya, 2003). Además, les permite ampliar su círculo de amistades, importante para mantener una red de apoyo.

Por otro lado, llama la atención que las parejas de las entrevistadas están de acuerdo con que salgan a trabajar fuera de casa, pero no se convierte en una condición para modificar la participación de estos en las tareas del hogar y la crianza de los hijos. Es decir, el que las mujeres se incorporen al campo laboral no necesariamente ha transformado, en lo sustancial, la división sexual del trabajo, siguen siendo ellas las principales responsables de las tareas domésticas y el cuidado de los hijos (Benería, 2006).

Existe una baja interferencia entre su vida personal y el trabajo, sin embargo, resulta necesario analizar bajo qué condiciones consiguen conciliar 
estos escenarios. Es decir, qué impacto tiene en otras áreas de su vida, como la salud física y mental, pues las tareas de casa demandan al menos 8 horas diarias de trabajo continuo (INEGI, 2014), generando una sobrecarga de trabajo relacionado con la tensión y agotamiento, de acuerdo a los estudios de Martínez et al. (2002).

Si tomamos en cuenta que cada vez más mujeres con responsabilidades familiares e hijos menores buscan trabajo fuera del hogar, se requiere prestar mayor atención a esta relación. Existe la necesidad de diseñar políticas de conciliación donde los sujetos de atención sean tanto hombres como mujeres, vigilando que estas acciones, como dice Faur (2006), no refuercen ideologías sexistas.

Queremos aclarar que no podemos circunscribir la equidad al hecho de la división de las tareas del hogar y el cuidado de los hijos, es un asunto complejo que involucra no solo la existencia de una ideología que condiciona un papel subordinado a las mujeres en relación con los hombres, sino también la prevalencia de prácticas de socialización, parcialmente ligadas al modelo hegemónico, que favorecen una desigualdad en el acceso a recursos, oportunidades y derechos a las mujeres. Construir la equidad en un contexto privado no se puede lograr sin tomar en cuenta cómo se encuentra la equidad en el nivel social, cultural e institucional, pues no radica en un trabajo meramente individual, sino que nos requiere a todas $y$ todos en los diferentes niveles.

\section{Referencias}

Benería, L. (2006). Trabajo productivo/reproductivo, pobreza y políticas de conciliación. Nómadas, 24, 8-21.

Bonino, M. L. (2004). Los micromachismos. La Cibeles, (2), 10-17.

Díaz-Guerrero, R. (2012). Psicología del mexicano. Descubrimiento de la etnopsicología. México: Trillas.

Díaz-Loving, R., Rivera, S., y Sánchez, R. (2001). Rasgos instrumental (masculinos) y expresivos (femeninos), normativos (típicos e ideales) en México. Revista Latinoamericana de Psicología, 33(2), 131-139.

Faur, E. (2006). Género, masculinidades y políticas de conciliación familia-trabajo. Nómadas, 24, 130-141.

Instituto Nacional de Estadística y Geografía - INEGI. (2012). Encuesta Nacional de Ocupación y Empleo 2012 (ENOE). Recuperado de http:// www.inegi.org.mx/est/contenidos/Proyectos/encuestas/hogares/regulares/enoe/

INEGI. (2013). Estadísticas a propósito del día de la mujer: datos de Coahuila. Boletín día de la mujer 2013. Recuperado de http://www.inegi.org.mx/inegi/contenidos/espanol/prensa/ Contenidos/estadisticas/2013/mujer5.pdf

INEGI. (2015). Estadísticas a propósito del día internacional de la eliminación de la violencia contra la mujer. Datos nacionales. Recuperado de http://www.inegi.org.mx/saladeprensa/aproposito/2015/violencia0.pdf

INEGI. (2014). Mujeres y hombres en México. Instituto Nacional de las Mujeres.

Martínez, C., Vera, J., Paterna, C., y Alcázar, A. (2002). Antecedentes del conflicto interrol y su relación con el esquema de género. Anales de Psicología, 18(2), 305-317.

Moya, M. (2003). El análisis psicosocial del género. En J. F. Morales y C. Huici (Eds.). Estudios de psicología social, (pp. 175-221). Madrid: UNED.

Organización para la Cooperación y el Desarrollo Económico - OCDE. (2015). Balance vida-trabajo. Recuperado de http://www.oecdbetterlifeindex.org/es/topics/work-life-balance-es/

Organización Internacional del Trabajo - OTI. (2016). Tendencias mundiales del empleo de las mujeres. Recuperado de http://www.ilo. org/public/english/employment/strat/download/getb07sp.pdf

Ortega, F. (1998). Imágenes y representaciones de género. Asparkía, (9), 9-20. 
Rocha, T. E., y Díaz-Loving, R. (2012). Identidades de género. Más allá de cuerpos y mitos. México: Trillas.

Secretaria del Trabajo y Previsión Social - STPS. (2016). Información laboral para Sonora. Subsecretaria de empleo y productividad laboral.

STPS. (2016). Información laboral para Coahuila. Subsecretaria de empleo y productividad laboral.
Tena, O., y Jiménez, P. (2006). Estrategias para mantener el modelo de masculinidad en padres-esposos desempleados. Revista de Estudios de Género. La ventana, (24), 440-462.

Tena, O. (2013). Estrategias utilizadas por mujeres policías para conciliar sus deberes de trabajo doméstico y remunerado: ¿un camino hacia la equidad? Acta Colombiana de psicología, 16(2), 81-91. doi: 10.14718/ACP.2013.16.2 